Ann. Sc. forest., 1978, 35 (1), 1-18.

\title{
Etude de la déviation des racines horizontales ou obliques issues de boutures de peuplier qui rencontrent un obstacle : applications pour la conception de conteneurs
}

\author{
A. RIEDACKER \\ Station de sylviculture ef de Production CNRF. INRA \\ Champenoux, 54280 Seichamps, France
}

avec la collaboration technique de J. F. MULLER ef J. M. GIORIA

\begin{abstract}
Résumé
Des études expérimentales ont montré que les racines issues de boutures de Peuplier rencontrant :

- un dièdre ne sont généralement déviées dans le sens de l'arête de ce dièdre que lorsque son angle est aigu,

- un obstacle court conservent après l'obstacle une direction de croissance proche de celle qu'elles avaient avant de rencontrer l'obstacle ;

- un obstacle long prennent une direction plus ou moins parallèle à celui-ci ;

- une succession d'obstacles, le dernier étant court et ne formant pas un angle aigu avec le précédent, prennent généralement une direction parallèle à celle qu'elles avaient avant de rencontrer le dernier obstacle.

Ces résultats peuvent fournir une interprétation quant à l'orientation des racines en milieu naturel ou en conteneurs. Ils peuvent servir de base à la conception de formes de conteneurs empêchant la spiralisation des racines latérales.
\end{abstract}

\section{Introduction}

La direction de croissance des racines est un des facteurs importants de la morphogenèse ; elle conditionne notamment l'exploration du sol ef la solidité de l'ancrage de la plante.

Dans la présente étude nous avons cherché à préciser le rôle que peuvent jouer des obstacles plans dans la détermination de la direction de croissance de racines non pivotantes.

Sur le plan pratique notre objectif était d'améliorer la forme des conteneurs afin d'éviter la spiralisation des racines latérales fréquemment observée sur des semis ou des boutures d'essences forestières ou d'ornement élevées en conteneurs souples (sachet de polyéthylène) ou rigides (Hiatt et Tinus, 1974 ; Franclet, 1975). Wilson (1967-1971) a montré précédemment que les racines latérales déviées dans le plan 
horizontal par un obstacle plat avaient une forte tendance à pousser, une fois franchi l'obstacle, dans le même sens qu'avant l'obstacle.

Dans les études qui suivent nous avons précisé l'effet :

- de la valeur de l'angle d'un dièdre formé par deux obstacles plans,

- de la longueur des obstacles,

- d'une succession d'obstacles,

sur la direction de croissance des racines latérales de boutures du Peuplier.

\section{Matériel et méthode}

Des boutures de Peuplier (Populus Xeuraméricana (Dode) Guinier et Populus trichocarpa Torr et Grey Cu FPL) élevées en serre développent rapidement des racines latérales à forte croissance radiale ; c'est la raison pour laquelle nous avons choisi ce matériel végétal. Des obstacles, en matière plastique PVC de forme et de longueur variables, ont été placés de diverses manières dans le milieu d'élevage constitué par un mélange de sable $(2 / 3)$ et de tourbe blonde broyée $(1 / 3)$. Les observations ont pu être faites généralement après 4 mois de végétation.

Nous appellerons, dans la suite du texte :

- «direction primitive » et « direction finale » les directions de croissance d'une même racine respeciivement avant et après un obstacle donné,

- «angle primitif vertical » et «angle final vertical» respectivement les angles que font les directions primitive et finale avec le plan horizontal ;

- «angle de déviation vertical » la différence entre la valeur de l'angle final vertical et l'angle primitif vertical ;

- «angle de déviation horizontal » l'angle que font les plans verticaux contenant respectivement la direction primitive et la direction finale;

- «angle primiłif (final) oblique », l'angle formé par la direction primitive (finale) et sa projection sur le plan de l'obstacle ;

- «angle de déviation oblique » la différence entre la valeur de l'angle final ohlique et de l'angle primitif oblique.

\section{Expérience $n^{0} 1:$ Influence d'un dièdre à arête verticale}

Pour préciser l'indicence de la valeur de l'angle des dièdres sur la déviation des racines nous avons réalisé des dièdres d'angle $\gamma\left(30^{\circ}, 45^{\circ}, 60^{\circ}, 90^{\circ}\right.$ et $\left.120^{\circ}\right)$ par pliage de plaques de PVC transparent de $1 \mathrm{~mm}$ d'épaisseur (fig. 1A et B).

Nous appellerons (fig. $1 \mathrm{C}$ et $\mathrm{D}$ ) :

$-\alpha$ l'angle primitif vertical exprimé en grades

- $\beta$ l'angle final vertical exprimé en grades.

Pendant l'expérimentation les racines étaient protégées de la lumière par du polyéthylène noir. Le cheminement des racines est reporté sur des feuilles de polyéthylène transparent ; lorsque l'on déplie ces dernières, les angles $\alpha$ et $\beta$ y sont directement mesurables. 


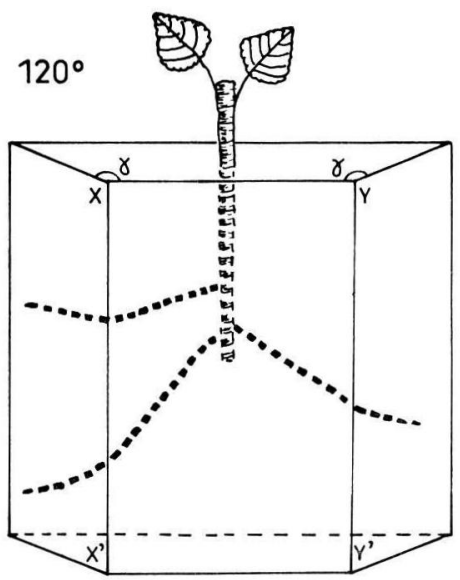

A

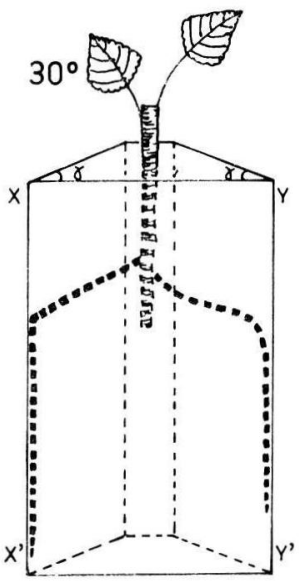

B

FIG. 1. - A et B : Représentations schématiques de boutures de peuplier placées dans des boîtes transparentes dont les parois forment des dièdres $(\gamma)$ de $120^{\circ}(\mathrm{A})$ et $30^{\circ}(\mathrm{B})$ d'arêtes $X X^{\prime}$ et $Y Y^{\prime}$.

A, B : Poplar cutting in transparent boxes with two dihedrals with angles of $120^{\circ}$ (A) and $30^{\circ}(B)$.

\section{Résultats}

Au moment du dépouillement nous avons regroupé les dièdres de $30^{\circ}$ et de $45^{\circ}$. Lorsqu'une racine rencontre un dièdre vertical de $120^{\circ}$, elle franchit généralement l'arête et continue à longer la deuxième face du dièdre ; l'angle de déviation horizontal est alors de $60^{\circ}$.

L'angle de déviation vertical est en revanche le plus souvent nul ou faible ; seulement 11 p. 100 des racines sont déviées verticalement par l'arête. Dans quelques cas cette déviation n'a lieu que sur 2-3 cm, l'angle final vertical étant alors très proche de l'angle primitif vertical ; ces cas particuliers se traduisant par un aspect en baïonnette n'ont pas été comptés avec les racines déviées dans le sens de l'arête (fig. $1 \mathrm{C}$ ).

TABLEAU 1

Comportement des racines rencontrant l'arête d'un dièdre

Behaviour of roots encountering a dihedral

Angle du dièdre

(Angle

of the dihedral) p. 100 de racines ne franchissant pas l'arête du dièdre

(p. 100 of roots which do not cross the intersection line of the dihedral) p. 100 de racines déviées dans le sens de l'arête

Nombre de racines

(p. 100 of roots becoming

parallel to the intersection (Number of roots) line of the dihedral

$\begin{array}{rrrr}120^{\circ} & 12 \text { p. } 100 & 11 \text { p. } 100 & 82 \\ 90^{\circ} & 40 \text { p. } 100 & 39 \text { p. } 100 & 74 \\ 60^{\circ} & 89 \text { p. } 100 & 75 \text { p. } 100 & 87 \\ 45 \text { et } 30^{\circ} & 98 \text { p. } 100 & 88 \text { p. } 100 & 171\end{array}$


$120^{\circ}$

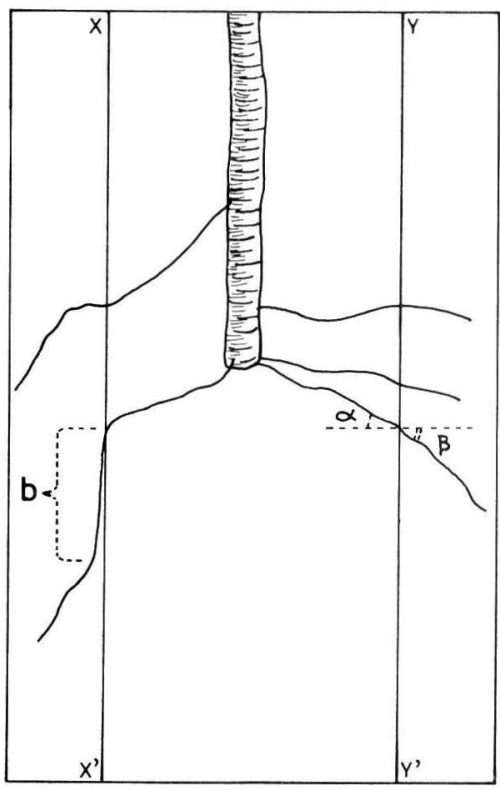

C $30^{\circ}$

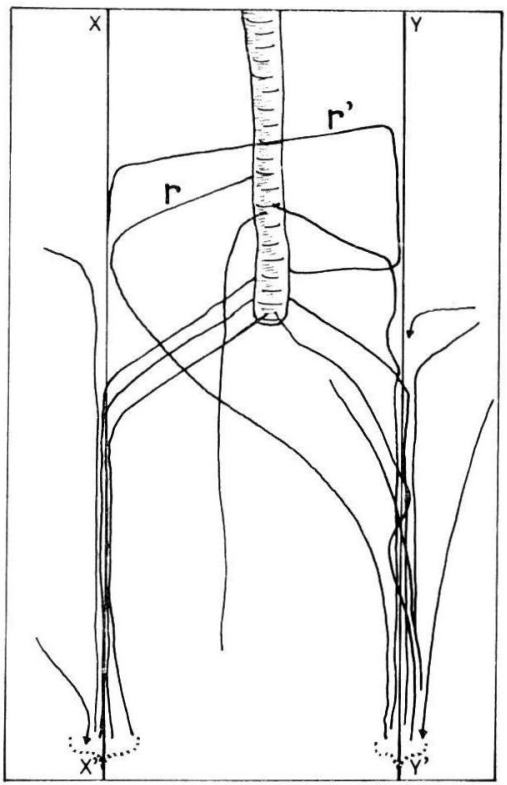

D

FIG. 1.

C et D : Schémas montrant le comportement des racines rencontrant l'arête d'un dièdre obtenus en décalquant les trajets des racines sur une feuille transparente appliquée sur les boîtes.

$\mathrm{C}=$ cas d'une boîte ayant deux angles dièdres de $120^{\circ}$

$\alpha$ : angle primitif vertical

$\beta$ : angle final vertical

b : effet baïonnette ; la racine n'est déviée suivant le sens de l'arête que sur une petite distance.

$\mathrm{D}=$ cas d'une boîte ayant deux angles dièdres de $30^{\circ}$

$r$ : exemple d'une racine qui, lorsqu'elle rencontre l'arête du dièdre dont l'angle est de $30^{\circ}$, ne la franchit pas et ne la suit pas.

$\mathbf{r}^{\prime}$ : exemple - (assez rare) - d'une racine déviée vers le haut et qui redevient horizontale après avoir longé l'arête (non comptabilisées avec les racines déviées dans le sens de l'arête).

C, D : Behaviour of roots encountering an obstacle.

C : Dihedral with angles of $120^{\circ}$

D : Dihedral with angles of $30^{\circ}$

$r=a$ root which does not follow the intersection line of the dihedral

$\mathbf{r}^{\prime}=$ a root which was deviated upwards and which became again horizontal (very rare).

Lorsque l'angle $\gamma$ du dièdre diminue le pourcentage de racines déviées dans le sens de l'arête augmente. Certaines racines (tabl. 1), surtout lorsque l'angle du dièdre est très aigu, sont même déviées de plus de 100 grades et reviennent en arrière sans franchir l'arête du dièdre (fig. 1 D). 

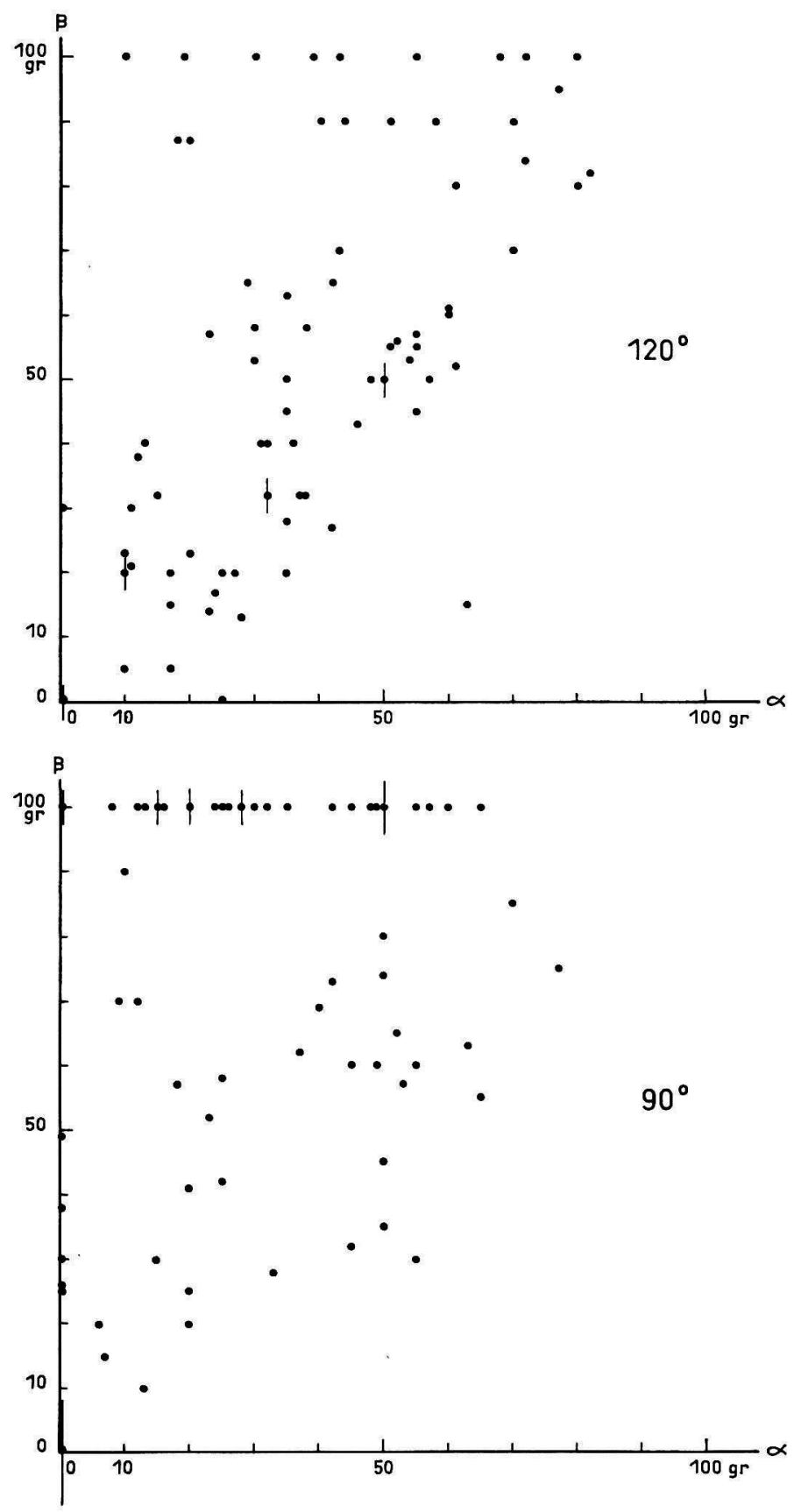

FIG. 2 A. 


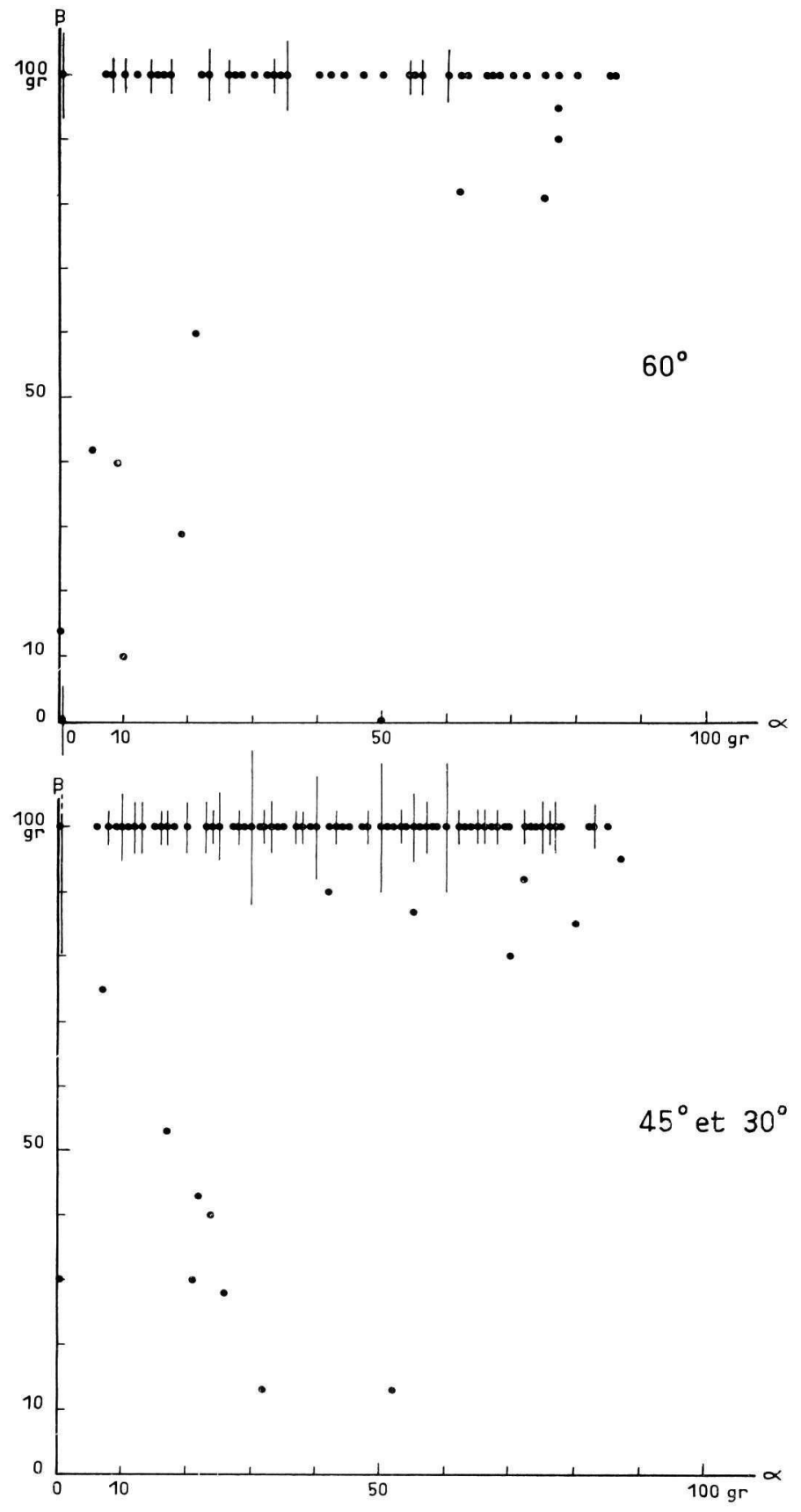

FIG. 2. - Déviation des racines rencontrant l'arête d'un dièdre dont l'angle $\gamma$ est de $120^{\circ}, 90^{\circ} ; 60^{\circ}$ ef $45^{\circ}$ ou $30^{\circ}$. On notera que lorsque l'angle du dièdre est de $120^{\circ}$, de nombreuses racines conservent la même pente après avoir franchi l'arête, tandis que lorsque l'angle du dièdre est de $60^{\circ}$, seules quelques racines ne sont pas déviées dans le sens de l'arête. La longueur du trait sur chaque point est proportionnelle au nombre de points figuratifs correspondant à une même situation.

Root deviation of roots encountering the intersection line of dihedrals with angles of $120^{\circ}, 90^{\circ}, 45^{\circ}$ or $30^{\circ}$. Notice that with an angle of $120^{\circ}$ many roots keep the same slope But only a few roots are not deviated vertically when the angle of the dihedral is only $60^{\circ}$ or $45^{\circ}$. 


\section{Expérience no 2 : Influence d'un obstacle plan vertical déviant une racine dans le plan horizontal}

Des obstacles plats, de longueur $(I=3,6,9,12 \mathrm{~cm})$ ont été placés tangentiellement à un cylindre fictif ayant pour axe la bouture et pour rayon $5 \mathrm{~cm}$ (fig. 3 ). Dans ces conditions, les racines attaquent ces obstacles avec un angle inférieur à $90^{\circ}$ et ne sont généralement déviées que dans le plan horizontal et non dans le plan vertical (cf. Expérience $n^{0} 1$ ) ; la longueur de la portion de racine comprise entre la bouture et le point de contact est dans ces conditions peu variable.

Nous appellerons (fig. 3 A et B) :

- $\alpha$ ' l'angle primitif oblique,

- $\beta^{\prime}$ l'angle final oblique,

- L la distance entre le point de contact de la racine avec l'obstacle et l'extrémité de l'obstacle,

- $\alpha^{\prime}-\beta^{\prime}$ l'angle de déviation oblique.

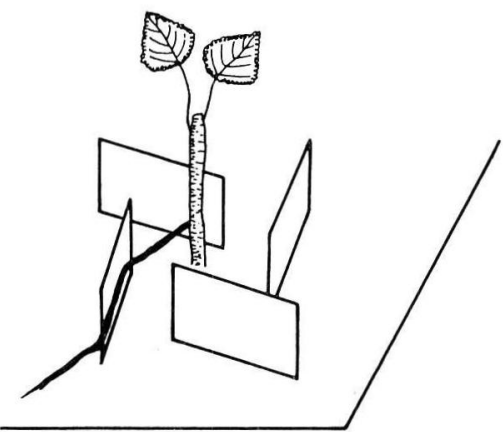

A

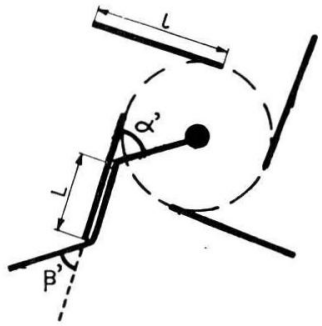

B

FIG. 3. - Représentation schématique de la disposition des obstacles plans verticaux autour d'une bouture.

(A) Vue en perspective $\alpha^{\prime}$ angle primitif oblique
(B) Vue de dessus $\beta^{\prime}$ angle final oblique

Experimental design for study of horizontal deviation of Poplar roots.

\section{Résultats : (fig. 4)}

Lorsque la longueur de la déviation est inférieure à environ $8 \mathrm{~cm}$, l'angle $\alpha^{\prime}$ a une valeur voisine de l'angle final $\beta^{\prime}$. La racine rencontrant un obstacle de moins de $8 \mathrm{~cm}$ de long se plaque contre celui-ci jusqu'à la fin de l'obstacle plan, puis se courbe vers l'extérieur «comme si » elle voulait conserver sa direction antérieure de croissance. L'angle de déviation oblique est nul ou très faible. 


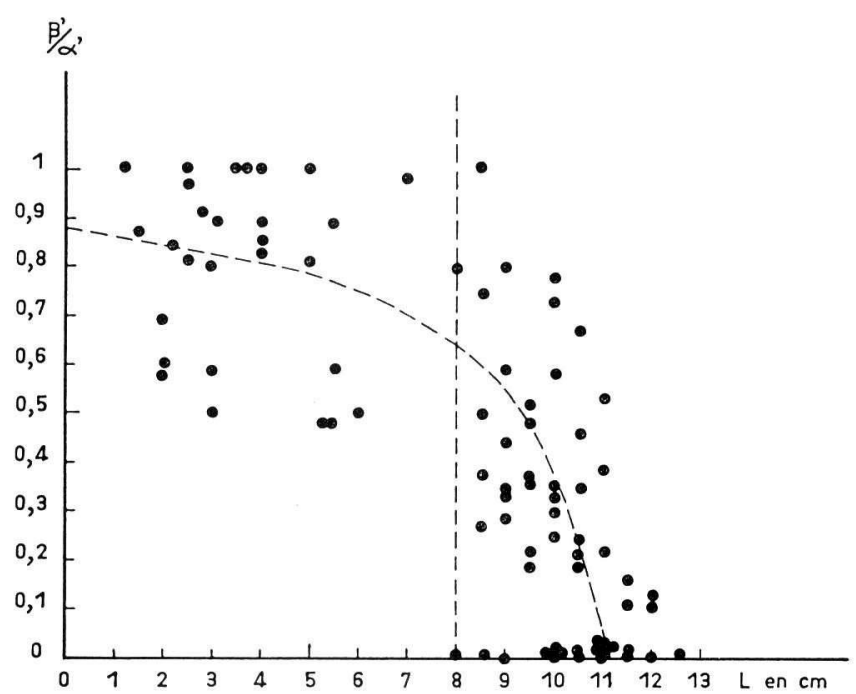

FIG. 4. - Influence de la longueur de déviation $L$ sur le rapport $\beta^{\prime} / \alpha^{\prime}$ lorsque la racine est déviée dans le plan horizontal.

Effect of the length of the deviation $L$ upon $\beta^{\prime} / \alpha^{\prime}$ when the roots are deviated horizontaly.

Lorsque la longueur de l'obstacle est supérieure à une dizaine de centimètres, la racine, en revanche, reste tantôł parallèle à l'obstacle, tantôt s'en écarte légèrement (fig. 8 B).

\section{Expérience no 3 : Influence d'un obstacle plan oblique déviant les racines dans le plan vertical}

Des obstacles plans ont été placés perpendiculairement à deux faces planes parallèles et verticales distantes de $2 \mathrm{~cm}$. L'angle $\delta$ que font ces obstacles avec le plan horizontal (fig. $5 \mathrm{~A}$ ) varie selon les essais entre $45^{\circ}$ et $135^{\circ}$. On se trouve ici dans une situa-
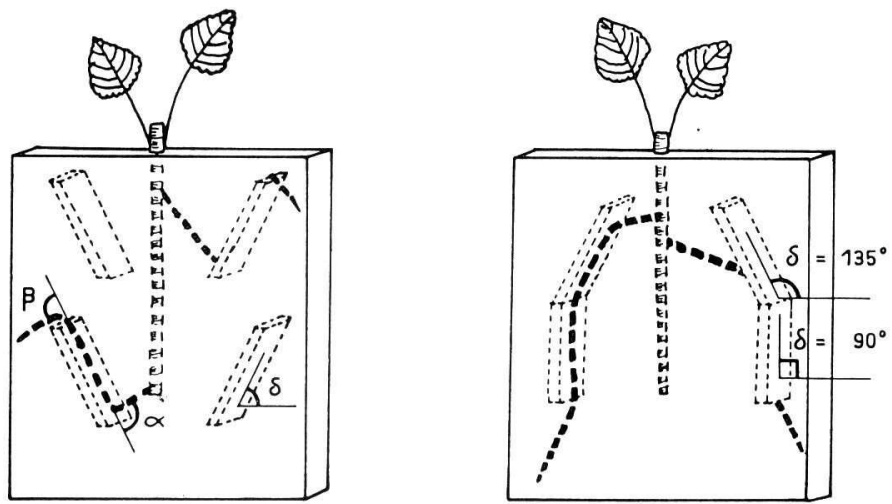

FIG. 5. - Représentation schématique des dispositifs destinés à dévier les racines dans le plan vertical. Experimental design for study of vertical deviation of roots. 
tion proche de l'expérience 2, à la différence cependant que la racine est contrainte à rester dans un plan vertical, du fait de la proximité des deux faces planes entre lesquelles elle est insérée ; ces faces peuvent presque être assimilées à celles d'un dièdre ayant un angle très aigu. La racine peut en revanche s'orienter librement dans le plan vertical.

\section{Résultats : (fig. 6)}

Comme précédemment, que l'obstacle soit oblique ou presque vertical, la racine longe l'obstacle qu'elle rencontre, puis reprend une direction finale ayant un angle final vertical peu différent de l'angle primitif vertical, à condition toutefois que la longueur de déviation ne dépasse pas $6 \mathrm{~cm}$.

Lorsque la longueur de déviation est supérieure à $7 \mathrm{~cm}$ l'angle final vertical dépend peu de l'angle primitif vertical, sauf peut-être pour les racines déviées vers le haut.

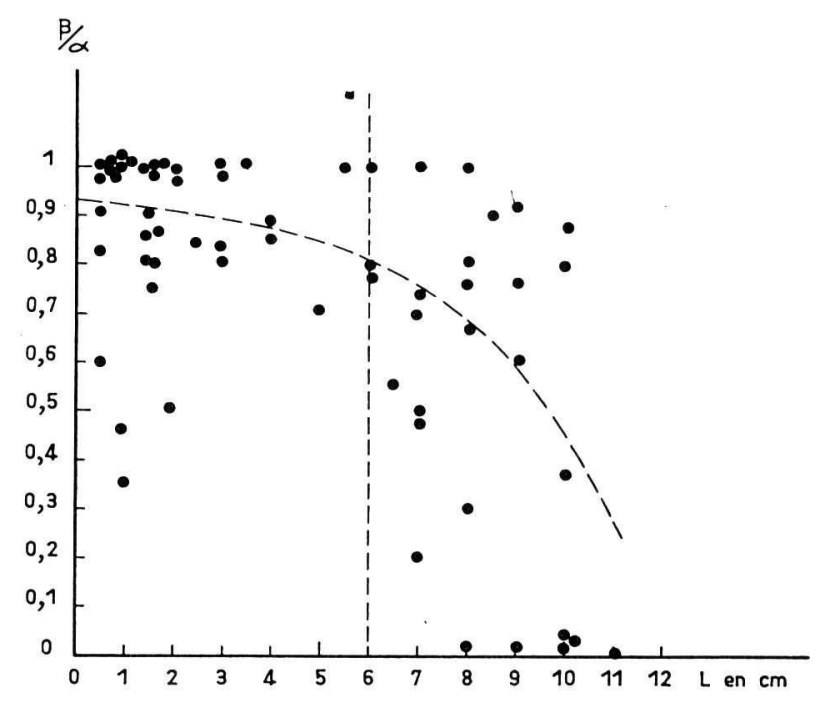

FIG. 6. - Influence de la longueur de déviation $L$ sur le rapport $\beta / \alpha$ lorsque la racine est déviée dans le plan vertical.

Influence of the deviation length $(L)$ upon $\beta / \alpha$ when the roots are deviated vertically.

\section{Expérience no 4 : Influence d'une succession d'obstacles}

Des obstacles de deux types provoquant plusieurs déviations ont été placés sur le cheminement des racines;

- d'une part (fig. 7) des obstacles constifués par une succession de dièdres à arête verticale et ayant un angle de $135^{\circ}$ (donc ne déviant généralement pas les racines dans le sens de l'arête) ; le premier plan de chaque obstacle multiple a une longueur de $12 \mathrm{~cm}$ ef les suivants une longueur de $3 \mathrm{~cm}$, 
- d'autre part (fig. 5 B) des obstacles constitués par des dièdres à arête horizontale.

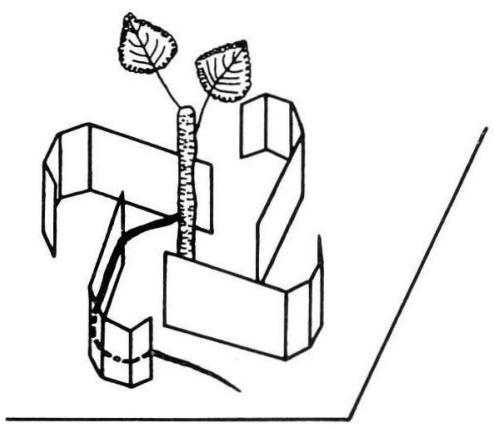

A

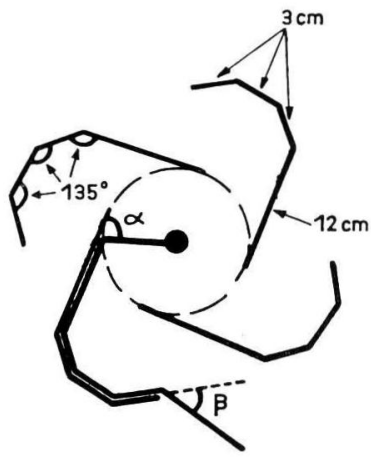

B

FIG. 7. - Représentations schématiques du dispositif destiné à dévier plusieurs fois une racine.

(A) Perspective; (B) Vue de dessus.

Experimental design for study of the effect of successive deviations of roots upon their final growth direction.

\section{Résultats}

Dans ces conditions la direction de croissance après le dernier obstacle a tendance à être parallèle à celle qu'avait la racine avant de rencontrer le dernier obstacle (fig. $8 \mathrm{C}$ ). Lorsque le nombre d'obstacles dépasse 3 ou 4, c'est-à-dire lorsque la somme des déviations imposées dans le plan horizontal, par les obstacles est supérieure à $90^{\circ}$ ou $135^{\circ}$, un grand nombre de racines plongent verticalement après avoir franchi deux ou trois arêtes.

\section{Conclusions et discussions}

Nous avons montré au cours de ces expériences qu'une racine de Peuplier rencontrant un obstacle qui le dévie de sa trajectoire primitive, longe cet obstacle sur une certaine longueur, puis, à condition que la longueur de la déviation ne dépasse pas 5 à $6 \mathrm{~cm}$, reprend une direction finale proche de sa direction primitive.

Lorsqu'elle rencontre une succession d'obstacles, la longueur du dernier étant courte (dans le cas présent $3 \mathrm{~cm}$ ), sa direction finale a tendance à être parallèle à sa direction avant son contact avec le dernier obstacle.

Ces résultats, qui s'appliquent à une déviation aussi bien dans le plan horizontal que dans le plan vertical montrent que la racine peut garder une certaine mémoire de sa direction de croissance antérieure, et qu'elle n'est pas déterminée par une action extérieure (points cardinaux par exemple) comme dans le géotropisme, puisque la direction finale, dans le cas où la racine rencontre une succession d'obstacles n'est pas déterminée par la direction primitive, mais par la direction avant la rencontre du dernier obstacle. 

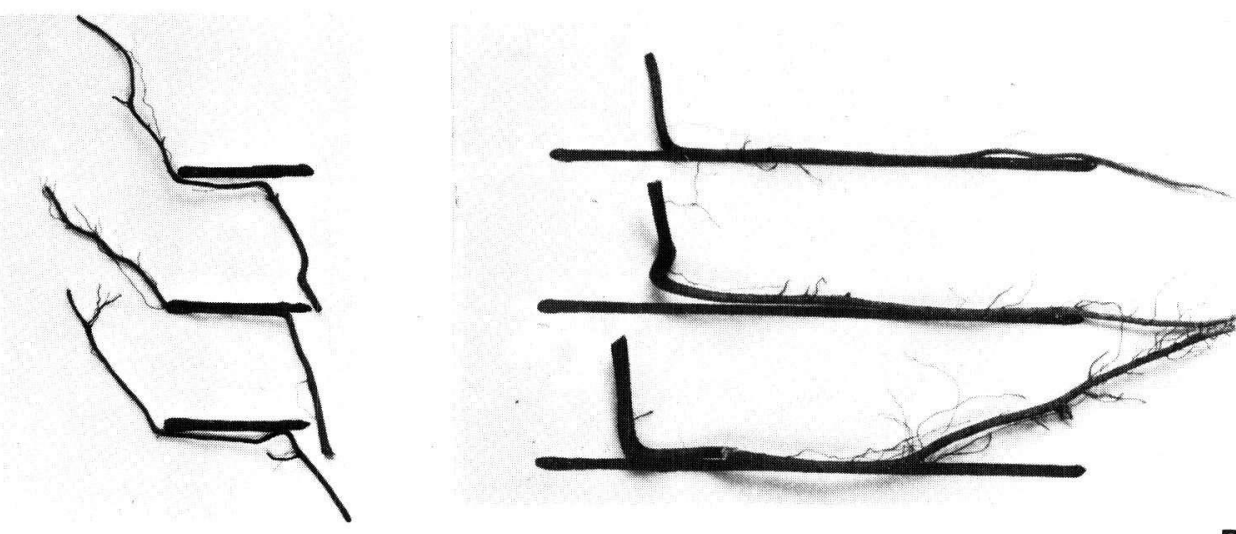

A

B
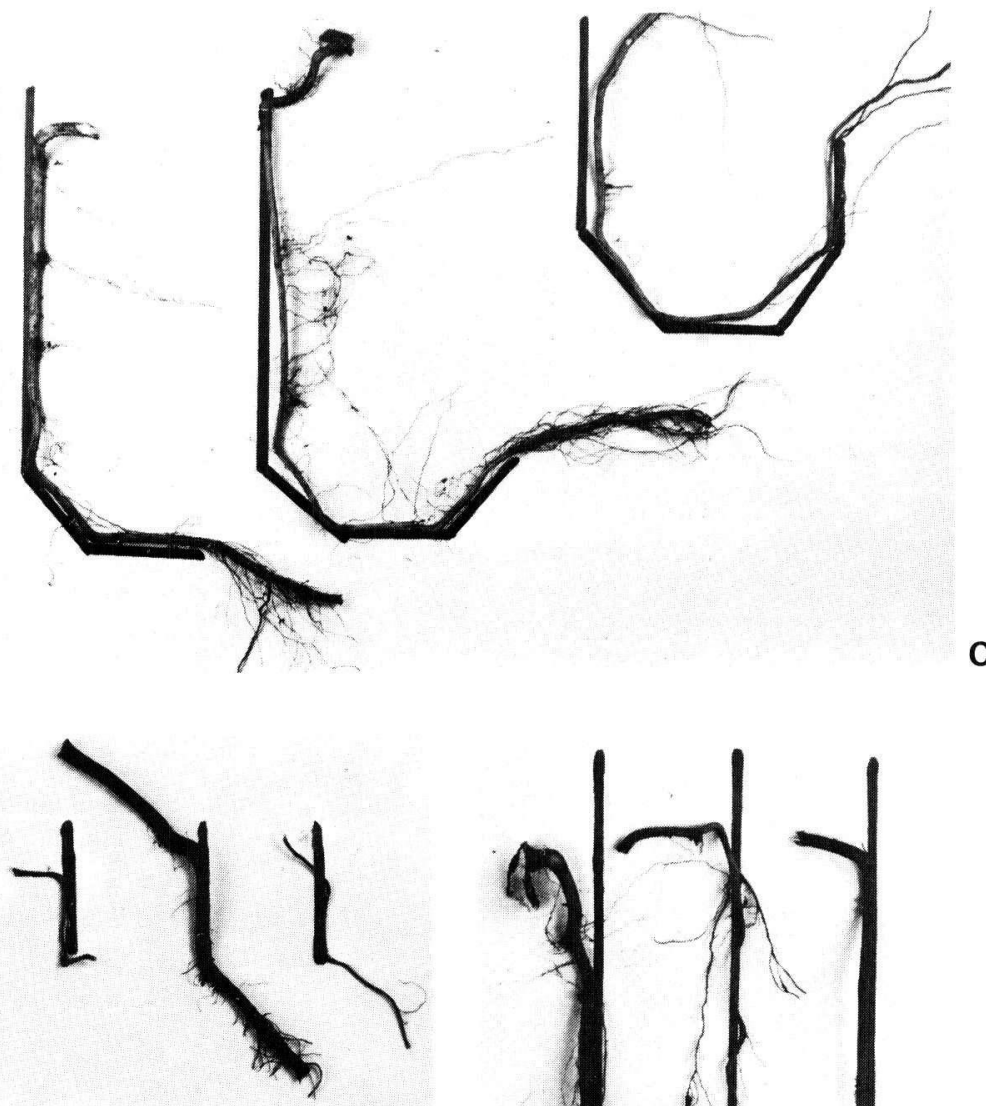

D

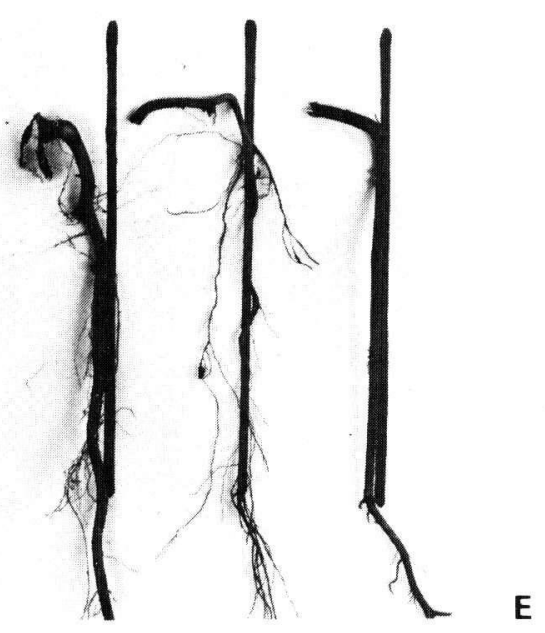

FIG. 8. - Photographie de racines ayant rencontré des obstacles plus ou moins longs dans le plan horizontal ( $A, B$ et $C$ ) et dans le plan vertical ( $D$ ef $E$ ).

Photography of roots which encountered obstacles of various lengths and which were deviated horizontally $(\mathrm{A}, \mathrm{B}, \mathrm{C})$ and vertically ( $\mathrm{D}$ and $\mathrm{E})$. 


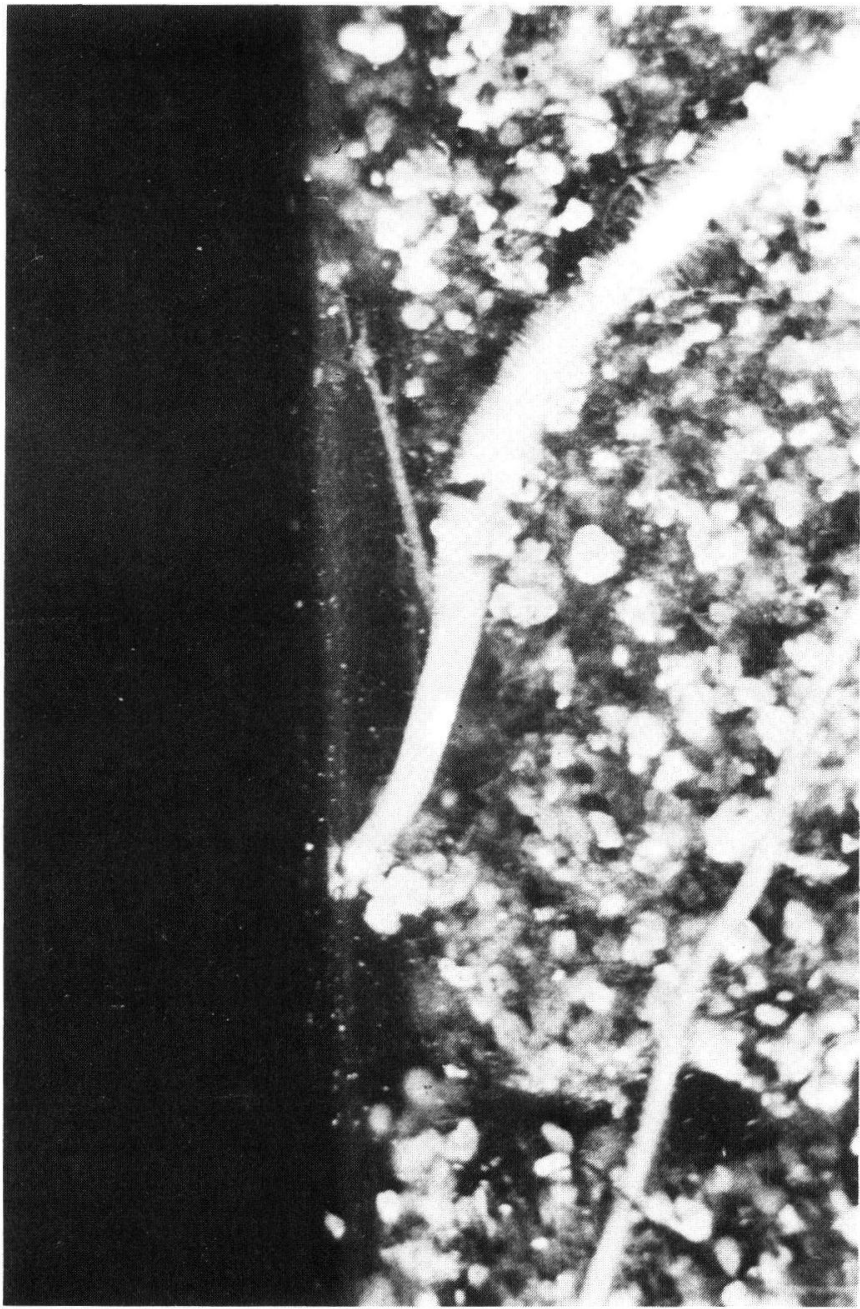

A

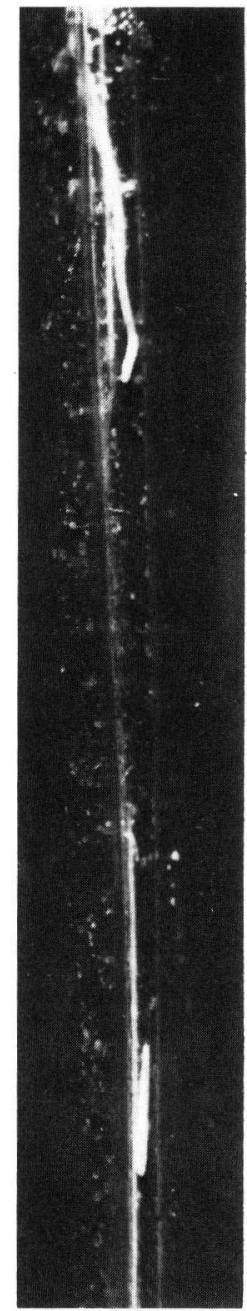

B

FIG. 9. - Aspects d'une racine rencontrant l'arête d'un dièdre dont l'angle est de $45^{\circ}$.

A : Vue de côté, à travers une des faces transparentes du dièdre, d'une racine déviée par l'arête du dièdre.

B : Vue frontale de racines "piégées » dans le coin de l'arête ; noter la position de la racine supérieure qui cherche à retrouver une position oblique.

Aspects of roots touching the intersection line of a transparent dihedral (450).

A : Side view of a root.

B : Front view of a root which «tries" to find again a horizontal growth direction.

Ces expériences pourraient permettre de supposer que les racines issues de boutures de Peuplier sont agéotropes. En réalité d'après les observations faiłes sur des racines en minirhizotrons ayant subi une rotation de $90^{\circ}$ dans le plan vertical, il apparaît que seules certaines racines sont réellement agéotropes, tandis que d'autres ne paraissent agéotropes que tant qu'elles n'ont pas subi de rotation les amenant 
dans une position au-dessus de l'horizontale ; lorsqu'elles font un angle* positif (sens trigonométrique) avec l'horizontale certaines racines se recourbent en effet vers le bas.

Une racine déviée vers le haut et faisant finalement un angle positif avec l'horizontale pourra se courber vers le bas à la fois à cause de sa direction dans l'espace et parce qu'elle a été déviée. Cela permet de comprendre pourquoi une racine déviée verticalement vers le haut peut redevenir subitement horizontale (fig. $1 \mathrm{D}$ racine $r^{\prime}$ ) alors que les racines déviées suffisamment longtemps vers le bas restent généralement verticales.

Certaines de ces conclusions ont déjà été partiellement formulées par Wilson (1964-1971) à la suite d'expériences faites avec des racines latérales d'Erables.

Cet auteur a notamment montré que la direction finale d'une racine après une déviation par un obstacle de l'extrémité d'une racine ou après un déplacement de toute la racine (ce qui, dans une certaine mesure, est comparable à la rotation que nous faisons subir aux plants) ne se traduisent pas par des réponses identiques ; après un déplacement vers le bas la racine poursuit généralement sa nouvelle direction de croissance alors qu'après une déviation elle se courbe et retrouve sa direction primitive ; en revanche après un déplacement de la racine au-dessus de l'horizontale celle-ci se recourbe vers le bas.

Quelles hypothèses peut-on formuler pour tenter d'expliquer la « mémoire » ou l'« autotropisme » (Troll, 1973) ou encore « la force longitudinale » (Rufelt, 1968) des racines de peuplier?

Selon Rufelt (1968) la «force longitudinale» serait l'une des trois composantes déterminant la direction de croissance d'une racine, les deux autres étant le géotropisme positif et le géotropisme négatif.

Ces mots ne permettent cependant pas d'avoir une connaissance plus approfondie des mécanismes.

Peut-être conviendrait-il d'entreprendre une étude histologique et peut-être verrait-on alors que la pointe de la racine "cherche » à retrouver la direction de croissance primitive tant que toutes les divisions cellulaires ne se font pas exclusivement suivant l'axe de déviation.

Bennet-Clark et coll. (1958) en stimulant latéralement l'extrémité d'une racine de haricot croissant verticalement dans l'air humide par un bref contact avec une toile d'émeri ou en collant une petite pièce de cette toile sur la racine pendant plusieurs heures ont constaté qu'on pouvait ainsi induire une courbure orientée vers le côté stimulé. Le simple contact avec un obstacle rugueux suffirait-il pour expliquer que la racine se courbe après l'obstacle?

Dans ce cas il conviendrait d'éviter d'employer les termes autopropisme et « mémoire» et de les remplacer par exotropisme comme le fit Wilson (1964).

Mais le fait que la racine puisse s'écarter de l'obstacle après une certaine longueur de déviation reste alors inexpliqué, de même d'ailleurs que le comportement des racines rencontrant un dièdre.

Lorsqu'une racine issue de boutures de peuplier rencontre :

- un dièdre vertical obtus, elle subit généralement une déviation importante dans le plan horizontal et faible dans le plan vertical, 
- un dièdre aigu, elle est généralement déviée suivant l'arête du dièdre, et cela avec une probabilité d'autant plus grande que le dièdre sera plus aigu.

Tout se passe comme si l'extrémité de la racine pouvait « explorer » avec plus ou moins de succès l'espace pour rechercher l'angle de déviation minimal. Divers auteurs ayant décrit des mouvements révolutifs de pointes de racines (Head, 1965 ; Fischer, 1964 ; Spurny, 1971), cette hypothèse ne paraît pas improbable et nous nous proposons de la vérifier ultérieurement. Sur la photographie 9 B on peut d'ailleurs observer que la racine supérieure déviée verticalement cherche à retrouver sa direction primitive.

\section{Conséquences pratiques}

Quelques enseignements peuvent être tirés de ces essais, tant pour l'observation des racines ef l'expérimentation que pour la pratique.

\section{Observation des racines}

L'observation des racines ayant un fort géotropisme peut être faite assez commodément en élevant les plants dans des minirhizotrons inclinés (Riedacker, 1974). Lorsqu'elles sont agéotropes et non issues d'une racine ayant un fort géotropisme, il est beaucoup plus difficile de les observer ; quand elles buttent sur la plaque transparente, elles ne la longent le plus souvent que sur une distance assez courte $(5 \mathrm{à} 15 \mathrm{~cm})$, après quoi elles peuvent disparaître dans le substrat du minirhizotron (fig. 10). On

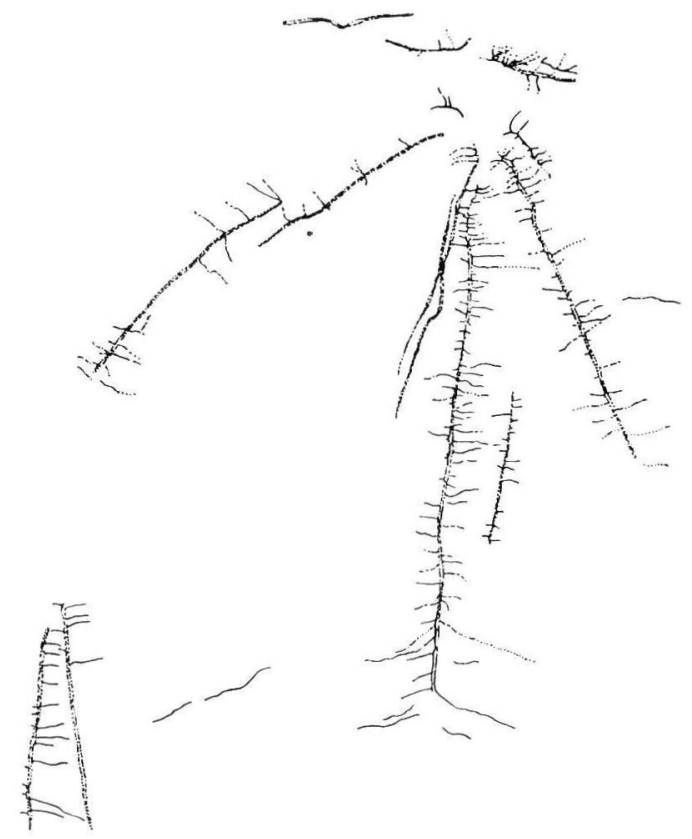

FIG. 10. - Système racinaire de peuplier noir issu de bouture à 5 mois. Observation à travers la face transparente d'un minirhizotron incliné (d'après Souidi, 1976). Echelle 2/9).

Root system of 5 month-old black poplar cutting as it can be observed through the transparent side of a minirhizotron (from Souidi 1976), Scale 2/9. 
peut remédier partiellement à cet inconvénient en donnant une forme semi-cylindrique au minirhizotron ; les pointes des racines buttant alors sur une distance beaucoup plus grande sur la face convexe restent plus longtemps apparentes. On pourrait également installer des déflecieurs, d'au moins $10 \mathrm{~cm}$ de large sur l'une des faces ou au centre, pour changer la direction de croissance des racines ef les renvoyer ainsi sur la ou les faces transparentes.

\section{Expérimentations sur les directions de croissance}

L'étude expérimentale de l'effet de la teneur en divers gaz de l'atmosphère du sol sur la direction de croissance des racines présente certaines difficultés, étant donné que les racines partent dans toutes les directions à partir de l'axe du plant. Cette étude montre qu'il est possible d'orienter la croissance des racines dans un même sens, à condition de les guider sur une distance suffisamment grande $(15 \mathrm{~cm})$; cette méthode permettrait donc de ne travailler que sur une étroite tranche de sol dans laquelle on pourrait plus facilement contrôler et faire varier les diverses conditions.

\section{Conteneurs évitant la formation de «chignons»}

Sur le plan pratique, cetle étude peut servir de base à la conception de conteneurs empêchant la spiralisation des racines latérales. Un conteneur en *M tel que celui de la figure 11 ayant quatre angles dièdres inférieurs à $60^{\circ}$ permet de dévier pratiquement toutes les racines latérales dans le sens des arêtes. Des résultats satisfaisants ont été obtenus avec des Hêtres et des Chênes; leurs racines latérales ne s'étaient, après un an, pas enroulées autour du pivoł (fig. 12) **.

La spiralisation du pivot ou des racines latérales dans le fond du conteneur, peut être évitée dans les conteneurs sans fonds posés sur un grillage ventilé par en dessous : les pointes des racines arrivant à l'air se déssèchent. L'intérêt majeur de cette forme en $M$, outre qu'elle évite la spiralisation, est qu'elle permet la juxtaposition des conteneurs sans perte de place (fig. 11).

Lorsque les plants ont un système racinaire puissant comme le Chêne ou le Hêtre, on peut donner une légère pente aux parois afin de faciliter le démoulage de la motte (fig. $11 \mathrm{D})$; on peut alors assembler les conteneurs à la manière des plateaux multipots scandinaves (Riedacker, 1976).

Mais pour pouvoir retirer sans risque des plants fragiles (semis de Cèdre par exemple) des conteneurs, il est absolument indispensable que ces derniers s'ouvrent latéralement, comme par exemple les conteneurs de la figure 11 ( $A$ et $B$ ) réalisés en deux parties I et II pouvant s'emboîter l'une dans l'autre et être facilement empilées pendant le stockage (fig. $11 \mathrm{C}$ ).

Lorsque les conteneurs pourront être réalisés avec une matière rapidement dégradable dans le sol, il ne sera plus nécessaire, ni de donner une pente aux parois, ni de pouvoir les ouvrir ; en revanche on pourra perforer les arêtes pour faciliter la sortie des racines (fig. $11 \mathrm{E}$ ).

* Modèle déposé.

** L'expérience no 1 a été reproduite avec des plants de Cèdre à la place de boutures de Peuplier ; comme précédemment la probabilité qu'une racine soit déviée verticalement augmente lorsque l'angle du dièdre diminue. 


\section{A. RIEDACKER}

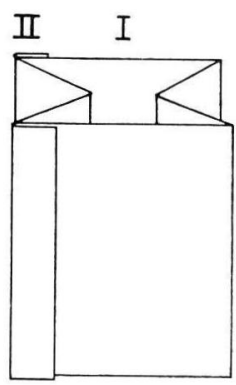

A

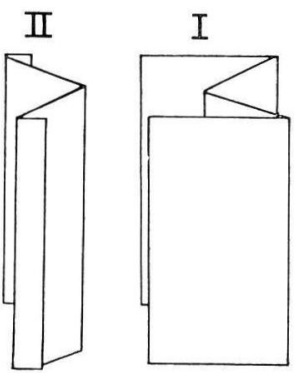

B
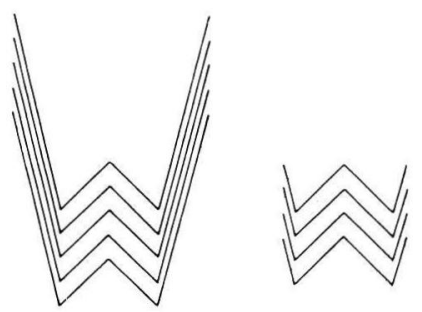

C

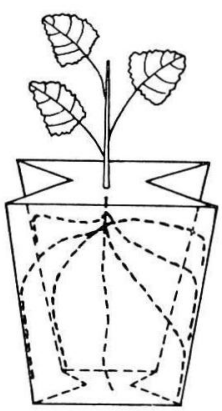

D

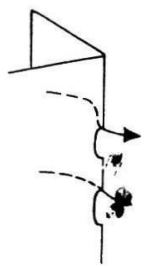

E

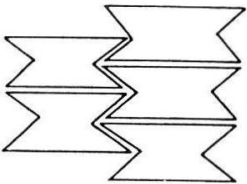

$\mathrm{F}$

FIG. 11. - (A et B) Conteneurs ouvrables en M ; la motte peut être extraite latéralement. (C) Stockage des deux parties des conteneurs ouvrants. (D) Conteneur $M$ non ouvrable à parois inclinées pour faciliter le démoulage. (E) Dièdre à arêtes perforées pour faciliter la sortie des racines. (F) Juxtaposition des conteneurs.

A, B : M container which can be opened (two parts). The plug can be easily extracted laterally. C : Stocking of the $M$ container. D : $M$ container in one piece which cannot be opened, but with sloping walls faciliting the removal of the plug. E : Dihedral with an interupted intersection line which allows the roots to come out. F : Container juxtaposition. 


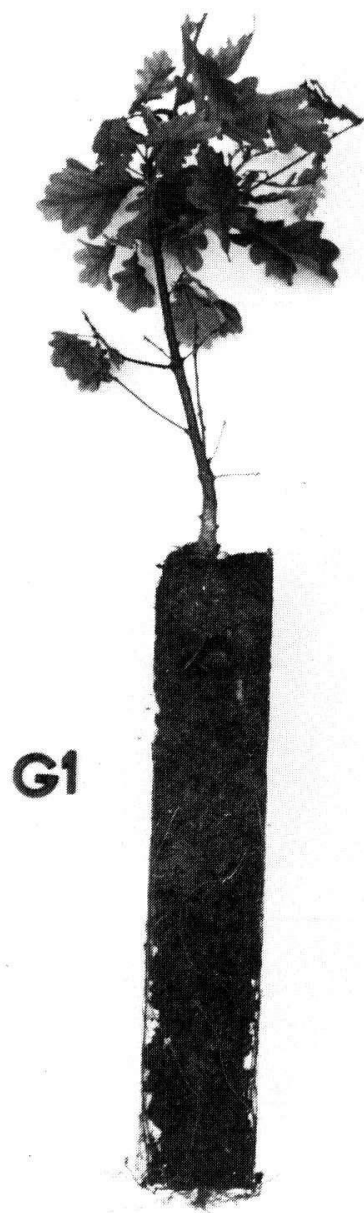

A

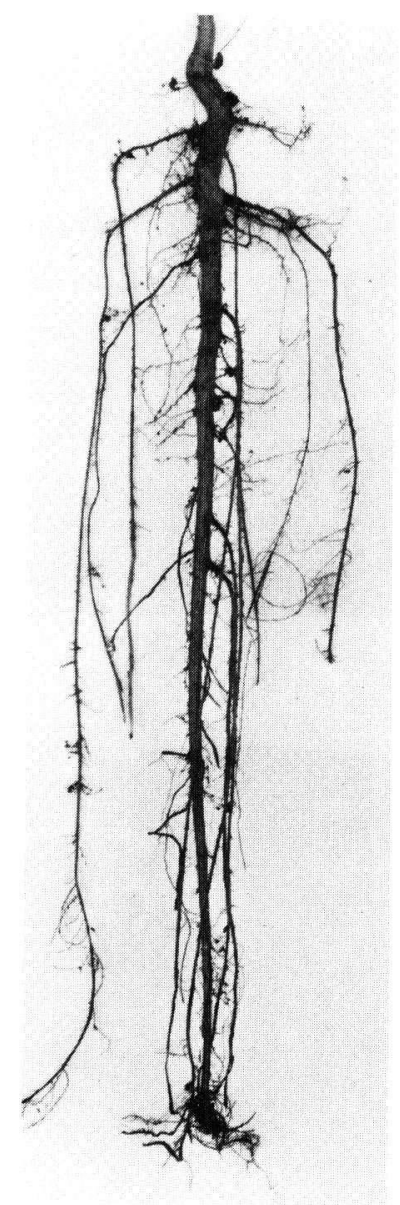

B

FIG. 12. - Plants de Chêne élevés en conteneurs $M$ de $40 \mathrm{~cm}$ de long.

(A) Aspect d'une motte en tourbe.

(B) Aspect d'un système racinaire.

Oak seedling raised in $M$ container $(40 \mathrm{~cm}$ long)

A : Aspect of the plug.

B : Aspect of the root system without peat.

Dans ces deux derniers cas les conteneurs seront nécessairement individualisés ce qui est extrêmement avantageux pour les semis de graines rares à germination irrégulière et pour le bouturage ; les conteneurs dans lesquels on n'aura semé qu'une graine et dans lesquels aucun plant ne se sera développé pourront être retirés et éventuellement être resemés ultérieurement.

II reste à préciser maintenant durant combien d'années on peut élever les diverses essences dans ces conteneurs et quelles conséquences cette technique nouvelle*

* Une note plus détaillée sur les différentes possibilités d'utilisation de ces conteneurs paraîtra dans la Revue Forestière Française. 
peut avoir sur la morphogenèse des systèmes racinaires après plantation, et de manière plus générale sur la reprise, la croissance et la stabilité des arbres ainsi élevés.

Reçu pour publication en décembre 1977.

\section{Summary}

Study of the deviation of horizontal or oblique roots of poplar cutting encountering an obstacle : Applications on container design

Experimental studies have shown that lateral roots from a Poplar cutting encountering :

- the intersection line of a dihedral, are generally deviated along this line only when the angle of the dihedral is acute ;

- an obstacle wich is short, keep their initial growth direction after the obstacle;

- an obstacle which is long, take finally a direction more or less parallel to the obstacle ;

- several obstacles at obtuse angles, the last obstacle being short, take finally a growth direction parallel to the penultimate obstacle.

The results may help to explain growth directions of roots in natural conditions and in containers A new container shape $(M)$ avoiding root coiling is also proposed.

\section{Références bibliographiques}

BENNET-CLARK T. A., YOUNIS A. F. et ESNAULT R., 1959. Geotropic behavior of roots. Jal. Exp. Bot., vol. $10, n^{\circ} 28$, pp. $69-86$.

FISCHER J. E., 1964. Evidence of circumnutational growth movements of rhizomes of Poa pratensis L. that aid soil penetration. Can. J. Bot., vol. 42, 293-299.

FRANCLET, 1975. Comment choisir un conteneur pour une production de plants forestiers ? Compte rendu des séminaires du groupe des racines, 23 oct. 1975, Grenoble, Tome 3, A. Riedacker et J. Gagnaire, pp. 12-35.

HEAD C., 1965. Studies of diurnal changes in Cherry root growth and nutational movements of Apple root tips by time lapse cinematrography. Annals of Bot. NS., vol. 29, no 114, 219-224.

HIATT H. et TINUS R., 1974. Container shape controls roots system configuration of Ponderosa Pine. Proceedings of the North American containerized Forest tree seedling symposium. Denver August 26-29 1974, Great Plains Agricultural Council, Publications no 68, 194-196.

RIEDACKER A., 1974. Un nouvel outil pour l'étude des racines ef de la rhizosphère ; le minirhizotron. Ann. Sc. For., 31 (2), 129-134.

RIEDACKER A., 1976. Production de plants d'Epicéa dans les pépinières norvégiennes. Revue Forestière Française, $\mathrm{n}^{\circ}$ 5, pp. 349-356.

RIEDACKER A., 1976. Les déformations racinaires. Compte-rendus des séminaires du groupe d'étude des racines. 5-6 oct. 76. Tome 4, $2^{\mathrm{e}}$ partie (Morphogenèse et mycorhizes). Problèmes posés aux pépiniéristes horticulteurs ef forestiers. A. Riedacker et J. Gagnaire E., Pp. 93-110.

RUFELT H., 1968. Geo and Hydrotropic responses of roots. In Root Growth Proceedings of the 15th Eastern School in Agricultural Science, pp. 54-64, Butterworths Londre.

SOUIDI C., 1976. Recherches physiologiques sur la croissance et la morphogenèse des racines chez Populus nigra et Picea exelsa. Thèse $3^{e}$ cycle Université de Grenoble, 102 p.

SPURNY M., 1971. Spiral oscillations resulting from a feedback control system in the growing pea root (Pisum sativum L.) Ecology and physiology of root growth. Internationales Symposium Potsdam September 1971, Akademie Verlag Berling, pp. 331-346.

TROLL, 1973. Allgemeine Botanik Lehrbuch Ferdinand Enke Verlag Stuttgard, p. 753.

WILSON B. F., 1967. Root growth around barriers. Bot. Gaz., 128, 79-82.

WILSON B. F., 1971. Vertical orientation of Red Maple (Acer rubrum L.) roots. Canad. Journ. For. Res., vol. $1,3,147-150$. 